\title{
Performance of Visual Tracking Systems: Implications for Visual Controlled Motion
}

\author{
N. J. Ferrier ${ }^{1}$ \\ Department of Mechanical Engineering \\ University of Wisconsin-Madison, WI, 53706 \\ Email: ferrier@engr.wisc.edu
}

\begin{abstract}
In visual control of motion the performance of the system is typically tied to the speed at which the visual information can be processed. Visual processing is unusual in that the data is not the information and in some sense the quality of the information can often be improved through extended processing (e.g. the resolution). A visual sensor tracking an object will only provide sporadic observations due to the processing delay, and, computational delay can pose difficulty within the control structure. We explore the trade off between the computational delay, the effective system observability, the accuracy of the visual processing, and implications for visual-guided motions. The task of visually tracking a target in order to localize it with sufficient accuracy to "touch" it provides a demonstration of the trade off between computational delay and the motion (visual localization) accuracy.
\end{abstract}

\section{Motivation}

When visual information is used to control motor systems, such as robots, control inputs for actuators are derived from the processing of image data. Typically the visual processing involves the extraction of contours, features, corners, or other visual primitives. Then, motion commands are determined by considering the image location of visual primitives, calibration information, and the desired robot task (see e.g. $[1,8,9,20,23,28])$. Tracking stability can only be achieved if the sensing delays are sufficiently small, and/or, if the dynamical model is sufficiently accurate. Various approaches have been taken to compensate for the delay of the visual system in visual servo control (see e.g. $[9,13,27,20]$ and other works on visual tracking $[4,22,25])$.

We consider a simple robotic reaching task: the robot must touch a moving target using target information derived from the processing of visual data. This task can be decomposed into the analysis of three separate

\footnotetext{
${ }^{1}$ This research supported in part by NSF IRI-9703352 0-7803-4394-8/98 \$10.00 ( 1998 IEEE
}

parts: (1) the target - the dynamics of the target including the system noise; (2) the vision system - resolution and accuracy of processing (measurement noise); (3) the motor system - speed and accuracy of the motor system and the visual-motor calibration. Of interest here is the relationship between the visual processing, its computational delay, and both the accuracy and resolution of the resulting target localization. For a particular manipulator, adequate tuning of the controller should enable motion to the commanded position with sufficient accuracy in time that depends only on the distance. Thus for a fixed distance to the target, any variation in success of reaching a target must depend on the accuracy of the commanded position (hence the visual system). Thus, with these assumptions, accuracy of the robot reaching task varies with the accuracy of the localization of the target by the visual system. The target properties effect the required resolution of the visual processing, which in turn effects the computation time. The uncertainty in the target dynamics influences the required resolution. The interplay of these factors for touching a static target was addressed in [12]. Here we further explore the interaction of the target dynamics, the resolution of visual data and give implications for the accuracy of reaching a moving target.

\section{Visual Tracking}

There has been considerable effort to produce real-time algorithms to track moving objects using visual sensors (CCD cameras). Various taxonomies of tracking algorithms are possible. If one classifies according to the type of feature tracked then we find methods to track points (see e.g. $[2,3,30]$ ), lines $[10,11]$, wire-frame models [17, 29], parameterized models [18] templates or regions [26, 21], and contours $[4,5,14,31]$. Except for certain implementations of contour tracking, most tracking algorithms utilize a focus of attention or region of interest. That is, information (features) is extracted from "sub-images" within the field of view. This paper considers only such "search window" or region of interest (ROI) based tracking. In order to restrict visual processing in this manner, the tracking system must 
have sufficiently rich prior knowledge of the target: either shape (or model-based) information, dynamic information, or both. Here we consider the use of target dynamic information. We shall demonstrate that the size and resolution of the ROI is of critical importance in visual tracking.

Often the visual tracking problem is formulated using a recursive filter framework [2, 4, 6, 22]. Generally, the system state represents the motion of the target. Even with the variety of features used for tracking, we can denote the location of the target in this manner (i.e. we can utilize the centroid or a particular feature of the target to denote the state) and we denote the state as $x(t)$. A stochastic model of the dynamics of the target is used:

$$
d x(t)=A x(t) d t+B d w(t)
$$

where $w(t) \sim N[0, Q]$. Under Gaussian noise assumptions, an estimate of the target, $\hat{x}$, and the associate covariance $P=E\left[(x-\hat{x}) \cdot(x-\hat{x})^{T}\right]$ are obtained using a Kalman-Bucy filter (see texts such as $[7,15,16]$ ). Accuracy of tracking is expressed via the covariance $P(t)$.

Although the target motion is continuous, the measurement process is discrete, not continuous. The visual system must perform image processing to locate the target position and hence measurements are available only after such processing has been completed. Next we consider separately variable processing time and fixed processing time visual tracking.

\subsection{Variable Processing Time}

Most tracking systems assume equally spaced measurements. Here we do not make that assumption in order to evaluate the effect of the search size on the tracking performance. Measurements are taken at discrete instances $\left(t_{1}, t_{2}, \ldots t_{k}, t_{k+1}, \ldots\right)$. The time of the measurements effects tracking stability. Historically there has been research on the optimal measurement schedule where the growth of the covariance is used to determine when to take measurements [19]. Due to the time taken for the processing of image data, the problem with computer vision is not determining how long to wait before taking a measurement, but determining how to measure often enough to ensure stability. Here we assume one finishes processing a previous measurement before taking the next measurement. The discrete measurement process is taken to be of the form

$$
y\left(t_{k}\right)=C_{k} \mathbf{x}\left(t_{k}\right)+v_{k}, \quad v_{k} \sim N\left[0, R_{k}\right] .
$$

The estimate of the state can be found from the state transition matrix on the interval $\left[t_{k}, t_{k+1}\right]$

$$
\hat{x}\left(t_{k+1}\right)=\Phi\left(t_{k+1}, t_{k}\right) x\left(t_{k}\right)=e^{A\left(t_{k+1}-t_{k}\right)} \hat{x}\left(t_{k}\right)
$$

and the covariance is determined (before measurement) from the Lyapanov equation ${ }^{1}$ :

$$
P\left(t_{k+1}^{-}\right)=e^{A\left(t_{k+1}-t_{k}\right)} P\left(t_{k}^{+}\right) e^{A^{T}\left(t_{k+1}-t_{k}\right)}+Q\left(t_{k}\right)
$$

where

$$
P\left(t_{k}^{+}\right)=\left(P^{-1}\left(t_{k}^{-}\right)+C_{k}^{T} R_{k}^{-1} C_{k}\right)^{-1}
$$

The covariance immediately before a measurement at time $t_{k+1}$ is given by:

$$
\begin{gathered}
P\left(t_{k+1}^{-}\right)= \\
e^{A\left(t_{k+1}-t_{k}\right)}\left(P^{-1}\left(t_{k}^{-}\right)+C_{k}^{T} R_{k}^{-1} C_{k}\right)^{-1} e^{A^{T}\left(t_{k+1}-t_{k}\right)}+Q\left(t_{k}\right)
\end{gathered}
$$

(where $Q\left(t_{k}\right)$ represents the integrated system noise on the interval $\left.\left[t_{k}, t_{k+1}\right]\right)$. We utilize this expression in determining the computational delay for tracking. If we assume that the measurement process takes time proportional to the size of the covariance (i.e. the ROI is determined based on $P\left(t_{k}^{-}\right)$). The time delay is

$$
t_{k+1}-t_{k}=\beta\left\|P\left(t_{k}^{-}\right)\right\|
$$

where || || denotes the "size" of the covariance (in pixels). For an image, the "size" is the number of pixels under consideration in a given algorithm (ROI). The parameter $\beta$ describes the processing time per pixel. Hence the total delay time is determined by the product of the search window size (the number of pixels to be processed) and the processing time per pixel. Obviously, if $P(t)$ grows then the process is effectively not observable - tracking is not possible. For multidimensional systems, solving equation 6 analytically may not be possible (see appendix $\mathrm{A}$ for a scalar example).

Consider the graphical representation of covariance for a state with two dimensions (figure 1). The ellipse represents the set of points

$$
\mathbf{x}^{T} P^{-1} \mathbf{x}=c
$$

The dashed lines represent the covariance before the image has been processed, $P\left(t_{k}^{-}\right)$. The size of the ellipse is the ROI which must be searched to ensure, with high probability, that the target will be located ${ }^{2}$. Under Gaussian assumptions, a " $2-\sigma$ " or " $3-\sigma$ " window is often used (hence $c=2$ or $c=3$ in equation 8 ).

After the processing has been completed (at time $t_{k+1}$ ), the covariance at time $t_{k}$ is represented by the grey

\footnotetext{
${ }^{1}$ We adopt the notation $P\left(t_{k}^{-}\right)$to denote the covariance at time $t$ prior to any measurement. In other notation this may be denoted $P\left(t_{k} \mid t_{k-1}\right)$. Likewise notations $P\left(t_{k}^{+}\right)$and $P\left(t_{k} \mid t_{k}\right)$ are equivalent.

${ }^{2}$ In image analysis one would most likely use the bounding rectangle of the ellipse to efficiently extract a ROI from a rasterscanned image. In the worst case scenario the bounding rectangle has sides proportional to the product of the eigenvalues of $P^{-1}$, hence the conservative $\|P\|$ operation would be of the form $\|P\|=$ $\Pi_{i}\left[\lambda_{i}\left(P^{-1}\right)\right]$ where $\lambda_{i}(A)$ denotes the eigenvalues of $A$.
} 


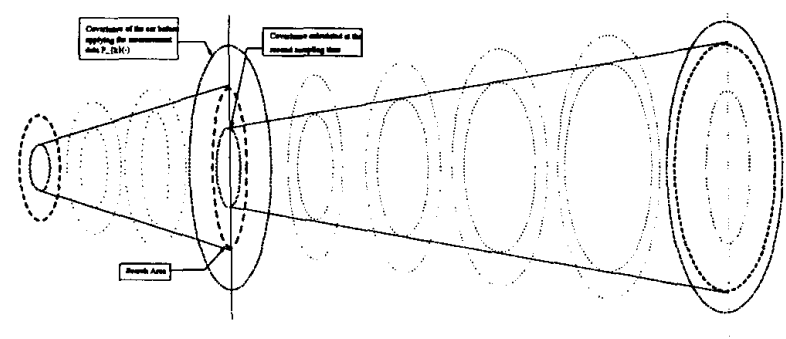

Figure 1: Graphic representation of the possible growth in state estimated covariance. If the delay is proportional to the search window size, which is proportional to the covariance, then the delay between measurements can grow, resulting in a system that is effectively unobservable.

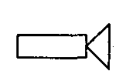

(a)

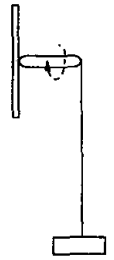

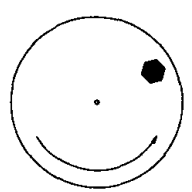

(b)
Figure 2: (a) Observing a target on a disk rotating with constant angular velocity (2nd order integrator). (b) Cartoon of the camera's view of the disk and target.

solid line. This covariance is used to estimate the current state covariance, $P\left(t_{k+1}^{-}\right.$using equation 6 . In the cartoon shown, the delay time is sufficiently large (for the given system dynamics and noise models) that the covariance at each measurement instant grows with time.

Example: Consider the simple example of tracking a target rotating at a constant angular velocity in a plane in front of, and parallel to, the image plane (see figure 2). The motion of the target can be expressed via a linear system (the second order integrator)

$$
\mathbf{x}=\left[\begin{array}{c}
\theta \\
\dot{\theta} \\
\ddot{\theta}
\end{array}\right], \quad \dot{\mathbf{x}}=\left[\begin{array}{ccc}
0 & 1 & 0 \\
0 & 0 & 1 \\
0 & 0 & 0
\end{array}\right] \mathbf{x}+\left[\begin{array}{c}
0 \\
0 \\
w
\end{array}\right]
$$

where $w \sim N\left[0, \sigma^{2}\right]$ is the system noise. Assuming the target can be readily located with standard visual processing (e.g. thresholding, connected component analysis and centroid computation, or, alternatively using a SSD approach) and that a camera calibration procedure has been performed, then the angle $\theta$ can be obtained from the image coordinates. The measurement process will be of the form

$$
y\left(t_{k}\right)=\left[\begin{array}{lll}
1 & 0 & 0
\end{array}\right] \mathbf{x}\left(t_{k}\right)+v_{k}, \quad v_{k} \sim N\left[0, r^{2}\right]
$$

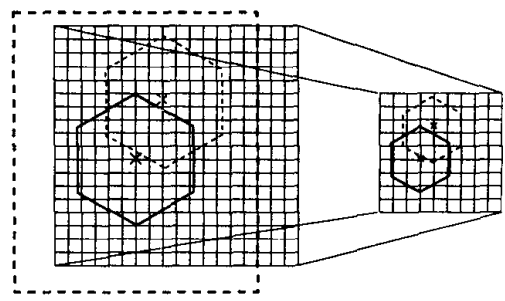

Figure 4: If the ROI gets too large then sub-sampling must be used to reduce processing time. This results in a decreased in the time delay, but increased measurement noise.

If one assumes a continuous measurement process then one can easily demonstrate that this system would be observable. However, in the visual tracking context, the continuous measurement assumption is not valid.

In the system given in equation (9), if the initial covariance is sufficiently small, and the processing time is sufficiently fast, then the target can be tracked. Figure 3 shows the estimated target position with the standard deviation of the position drawn with dashed lines. The graphs on the right show the identical system, however the image processing time $(\beta)$ is doubled. The lower graphs show the delay time between measurements. For the equivalent time ( $5 \mathrm{~s})$, the system shown on the right (with slower image processing) performs only five measurements, while the faster processing system performs sixteen measurements. Notice that the covariance (and hence the image processing delay time) reaches a steady state for the system with faster processing. For the system with slower processing, the delays increase monotonically after the 2nd measurement. This system is unable to track the target.

\subsection{Fixed Processing Time}

With the exception of asynchronous frame grabbers, most images are acquired at a fixed rate and real-time vision algorithms attempt to process at that rate (or some multiple of that rate). In order to achieve frame rate performance, the processing time can be decreased by either, 1 . decreasing the processing time per pixel $(\beta$ in equation (7)) or 2 . processing fewer pixels by decreasing the size of the ROI, or sub-sampling the ROI. While the increasing speed of processors, and often some clever programming can achieve the first alternative, there will always be a limit to processing time reductions on any given processor. Under the assumption of a particular hardware set-up and reasonably written code, here we consider reductions in the number of pixels to be processed. Sub-sampling has been used previously to ensure a fixed imaging delay [24], however no analysis was performed to determine the effect of this sub-sampling on system performance. 

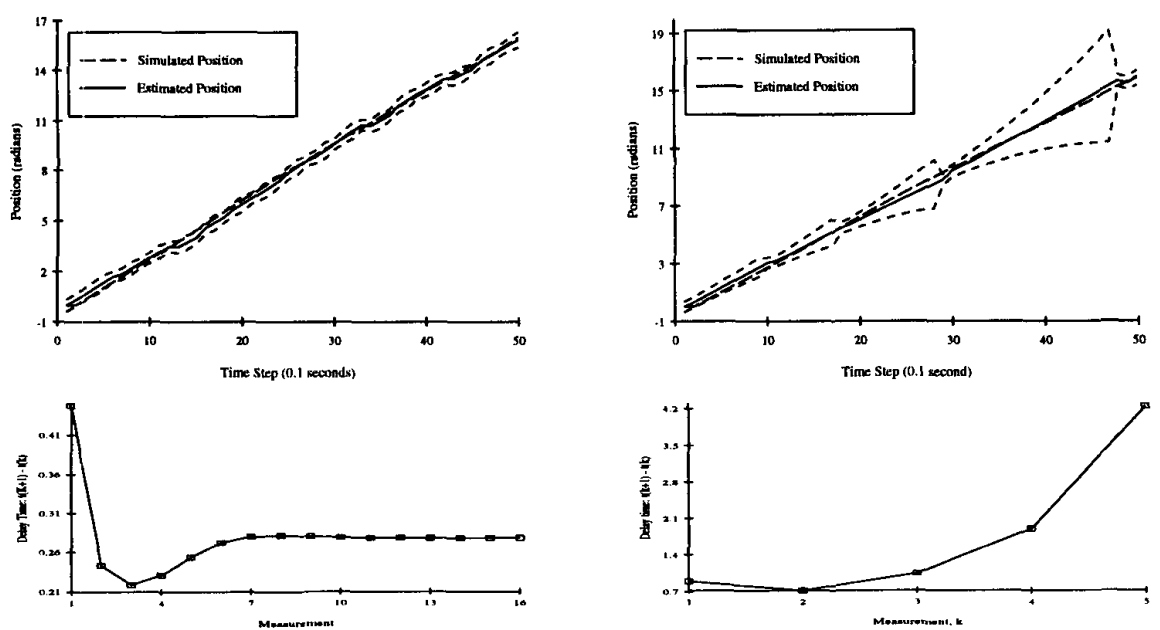

Figure 3: Estimated position of target with dashed lines showing the position covariance. Right hand side shows doubled processing time. (Initial speed is $33 \mathrm{rpm}$, system noise is $N\left[0,0.04 \mathrm{rad} / \mathrm{s}^{2}\right]$ ).

ROI-based tracking will succeed or fail based on the growth of $P$. $P$ evolves according to the Riccati equation given in equation (6). Note that the growth of the covariance depends on the dynamics, $A$, and the time interval $\left(t_{k+1}-t_{k}\right)$. Solving equation 6 analytically can only be performed in special cases (see e.g. Gelb [15], chapter 4 , example 1 which solves the Riccati equation for a second-order integrator with no system noise and appendix A which considers a scalar system).

The tracking example given earlier (equation 9 and figure 3) can be stabilized (the time intervals held fixed) by sub-sampling the ROI. A fixed sub-sampling (as done in [24]) does not necessarily solve the problem. In figure 5(a), the position covariance of our example system with slow processing and sub-sampling is shown. While sub-sampling achieves improved performance, the time delays still increase monotonically.

To hold the time delay constant, (assuming a given tracking algorithm, i.e. fixed processing speed, $\beta$ ), we must sub-sample the ROI by some factor denoted $\alpha$ :

$$
t_{k+1}-t_{k}=\beta\left\|\alpha P\left(t_{k}^{-}\right)\right\|=\text {constant }
$$

(for $0<\alpha<1$ ). The resolution (measurement covariance) of the measurement process then increases by $1 / \alpha$. In figure $5(\mathrm{~b})$, the resolution is actively controlled to ensure that the time delay remains below some threshold. The slow tracking algorithm using resolution control reaches a "steady state". In the figure shown, our constant time interval $t_{k+1}-t_{k}$ is not a multiple of the the discrete time sampling, hence the covariance "steady-state" oscillates between two values. The "steady-state" covariance is larger than the equivalent dynamic system with fast processing (solid line in figure 5). However, the system with slow processing can now track the target, but with larger uncertainty.

\subsection{Metrics to assess performance}

In the appendix, an analytic analysis of variable resolution tracking in one dimension is given. For the scalar system once can give an explicit relationship between the resolution, the dynamics and the time delay. Analytic results in higher dimensions are difficult to obtain, instead we us consider metrics to assess performance during computation. If the processing time $(\beta)$ for an algorithm has been determined and the delay time fixed, on-line monitoring of the covariance $P$ allows us to use equation 10 to determine the sub-sampling factor.

For continuous-discrete systems observability depends on the information matrix [16]

$$
\mathcal{I}\left(t_{k}, t_{j}\right) \triangleq \sum_{i=j}^{k} \Phi^{T}\left(t_{k}, t_{i}\right) C^{T}\left(t_{i}\right) R^{-1}\left(t_{i}\right) C\left(t_{i}\right) \Phi\left(t_{k}, t_{i}\right)
$$

for $t_{k}>t_{j}$. In order to determine $x_{k}$ from measurements $y_{j}, \ldots, y_{k}, \mathcal{I}\left(t_{k}, t_{j}\right)$ must be positive definite. The interval over which we compute the information matrix effects the singularity of $\mathcal{I}$. Hence any definition of observability of the system must consider the interval for which we are computing $\mathcal{I}$. In practice, the non-singularity of the information matrix is of less importance than its condition number. Systems with information matrix with high conditions numbers are effectively non-singular. The change of the condition number provides feedback to alert the system of tracking difficulties. 


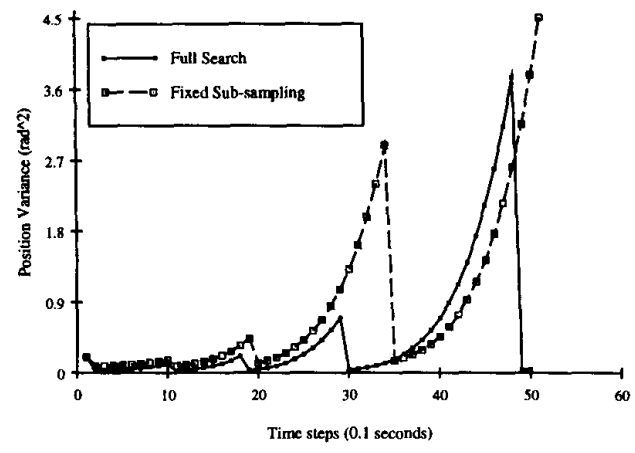

(a)

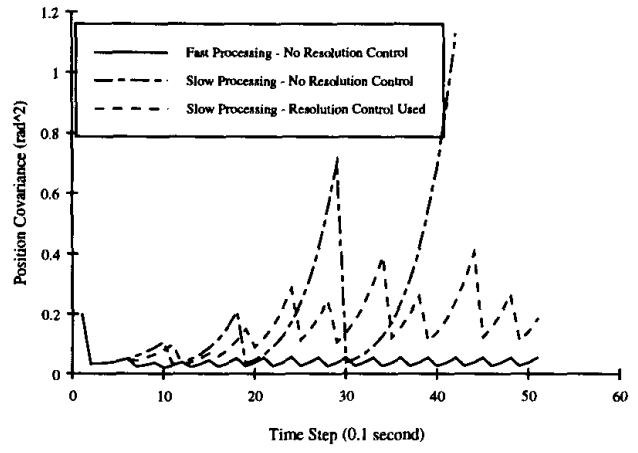

(b)

Figure 5: (a) The covariance for target position shown for full search and sub-sampling. Even with sub-sampling the covariance can grow monotonically. (b) Graph comparing target position covariance for a fast algorithm, a slow algorithm with no resolution control and a slow algorithm using resolution control. Notice that the slow algorithm with resolution control reaches a steady state. The "steady-state" covariance is larger than the equivalent dynamic system with fast processing.

\section{Discussion: Implications for Visual Robot Control}

For a given target speed, visual tracking can produce an estimate of the target position (at a particular accuracy and resolution). If the images processed asynchronously (completing processing of one image before acquiring the next), then visual tracking can become unstable. To process the images as they arrive (at say, $30 \mathrm{~Hz}$ for NTSC standard) the either the ROI size must be adjusted (risking tracking failure) or the ROI must be sub-sampled. The visual system commands the motion system (which requires calibration between the vision and the motor system - see e.g. $[8,27])$. The speed with which motors move, along with the delay due to visual processing can be accounted for in the motor control system. If the dynamics of the target are such that a resolution control scheme must be invoked, then the resulting accuracy of the robot motion may be compromised. For simple target motion, we provided examples of expected system behavior for slow vs. fast image processing. In static reaching tasks, multiple motions may be used to successfully reach the target [12], however for a moving target the resolution of the target location may be insufficient for robot manipulations. One can quantify both the accuracy of the tracking system (via the covariance) and the observability of the tracking system (via the information matrix). On-line monitoring of the size of the covariance (or the condition number of the information matrix) provides metric information for determining 1) what resolution to use and 2) whether to attempt to use the visual information for robot control.

\section{References}

[1] P. Allen, B. Yoshimi, and A. Timcenko. Real-time visual servoing. In Proc. IEEE Int. Conf. Robotics and Automation, volume 1, pages 851-856, 1991.

[2] N. Ayache and O. D. Faugeras. Maintaining representations of the environment of a mobile robot. In Int. Journal of Robotics Research. MIT Press, 1987.

[3] P. Beardsley and A. Zisserman. Affine structure from motion. In Proc. 4th Int. Conf. on Computer Vision, 1995.

[4] A. Blake, R. Curwen, and A. Zisserman. A framework for spatio-temporal control in the tracking of visual contours. Int. Journal of Computer Vision, 1993.

[5] A. Blake and M. Isard. Active Contours. Springer Verlag, 1998.

[6] T. J. Broida and R. Chellappa. Estimating the kinematics and structure of a rigid object from a sequence of monocular images. IEEE Transactions on Pattern Analysis and Machine Intelligence, 13(6):497-512, June 1991.

[7] A. Bryson and Y. Ho. Applied Optimal Control. Hemisphere Publishing Corp., New York, 1975.

[8] A. Castano and S. Hutchinson. Visual compliance: Task-directed visual servo control. IEEE Transactions on Robotics and Automation, 10(3):334-342, 1994.

[9] J. Clark and N. Ferrier. Modal control of visual attention. In Proc. of the Int'l Conf. on Computer Vision, pages 514-531, Tarpon Springs, Florida, 1988.

[10] R. Deriche and O. Faugeras. Tracking line segments. In O. Faugeras, editor, Proc. 1st European Conference on Computer Vision, pages 259-268. Springer-Verlag, 1990.

[11] O. Faugeras, F. Lustman, and G. Toscani. Motion and structure from motion from point and line matches. In Proc. Int. Conf. on Computer Vision, pages 25-34, 1987.

[12] N. Ferrier. Achieving a Fitts law relationship for visual guided reaching. In Proc. 1998 IEEE International Conf. on Computer Vision, Mumbai, India, 1998. 
[13] N. Ferrier and J. Clark. The Harvard Binocular Head. Int'l Journal of Pattern Recognition and AI, pages 9-32, March 1993.

[14] N. Ferrier, S. Rowe, and A. Blake. Real-time traffic monitoring. In IEEE Workshop on Applications of Computer Vision, Sarasota, FL, December 1994.

[15] A. Gelb and the Technical Staff, Analytic Sciences Corporation. Applied optimal estimation. MIT Press, Cambridge, MA, 1974.

[16] A. Jazwinski. Stochastic processes and filtering theory. Academic Press, 1970.

[17] D. Koller, D. Danilidis, and H.-H. Nagel. Modelbased object tracking in monocular image sequences of road traffic scenes. Int. Journal of Computer Vision, 10(3):257$281,1993$.

[18] D. Lowe. Robust model-based motion tracking through the integration of search and estimation. Int. Journal of Computer Vision, 8(2):113-122, 1992.

[19] R. Mehra. Optimization of measurement schedules and sensor designs for linear dynamic systems. IEEE Transactions on Automatic Control, 21(1):55-64, 1976.

[20] B. Nelson, N. Papanikolopoulos, and P. Khosla. Visual servoing for robotic assembly. In Visual Servoing-RealTime Control of Robot Manipulators Based on Visual Sensory Feedback, pages 139-164. World Scientific Press, 1993.

[21] K. Nichols and S. Hutchinson. Weighting observations: the use of kinematic models in object tracking. In Proc. 1998 IEEE International Conf. on Robotics and Automation, 1998.

[22] C. Olivier. Real-time observatiblity of targets with constrained processing power. IEEE Transactions on $\mathrm{Au}$ tomatic Control, 41(5):689-701, 1996.

[23] K. Pahlavan and J.-O. Eklundh. Heads, eyes and head-eye systems. In H. Christensen, K. W. Bowyer, and H. Bunke, editors, Active Robot Vision. World Scientific Press, 1993.

[24] N. Papnikolopoulos, P. Khosla, and T. Kanade. Visual tracking of a moving target by a camera mounted on a robot: A compbination of control and vision. IEEE Transactions on Robotics and Automation, 9(1), 1993.

[25] J. Roberts and D. Charnley. Parallel attentive visual tracking. Engineering Applications of Artificial Intelligence, $7(2): 205-215,1994$.

[26] S. Scarloff. Active blobs. In Proc. 1998 IEEE International Conf. on Computer Vision, pages 1146-1153, Mumbai, India, 1998.

[27] T. Schnackertz and R. Grupen. A control basis for visual servoing tasks. In Proc. 1995 IEEE Conf. on Robotics and Automation, Nagoya, Japan, 1995.

[28] J. Son, R. Howe, J. Wang, and G. Hager. Preliminary results on grasping with vision and touch. In Proceedings of the IEEE Int. Conference on Intelligent Robots and Systems, 1996.

[29] G. Sullivan. Visual interpretation of known objects in constrained scenes. Phil. Trans. R. Soc. Lond. B., B(337):109-118, 1992.

[30] P. Torr, A. Fitzgibbon, and A. Zisserman. Maintaining multiple motion model hypotheses over many views to recover matching and structure. In Proc. 1998 IEEE International Conf. on Computer Vision, pages 485-491, Mumbai, India, 1998.
[31] A. Yuille, D. Cohen, and P. Hallinan. Feature extraction from faces using deformable templates. In Proc. CVPR, pages 104-109, 1989.

\section{A. 1D Analytic Results}

We present a $1 \mathrm{D}$ example analytically. The following example follows closely from that given in [22]). The one dimensional linear system:

$$
\begin{aligned}
d x & =a x d t+d w ; E\left[d w^{2}\right]=\sigma^{2} d t \\
y\left(t_{k}\right) & =x\left(t_{k}\right)+v ; v\left(t_{k}\right) \sim N\left[0, R_{k}\right]
\end{aligned}
$$

For this scalar system equation 6 is

$$
P\left(t_{k+1}^{-}\right)=e^{2 a\left(t_{k+1}-t_{k}\right)} \frac{P\left(t_{k}^{-}\right) R_{k}}{P\left(t_{k}^{-}\right)+R_{k}}+\frac{\sigma^{2}}{2 a}\left(e^{2 a\left(t_{k+1}-t_{k}\right)}-1\right)
$$

This general form of $P\left(t_{k+1}^{-}\right)$can be shown to grow for particular dynamics and measurement noise. Note that if

$$
t_{k+1}-t_{k}>\frac{1}{2 a} \log \left(\frac{P+R}{R}\right)
$$

(where we abbreviate $P=P\left(t_{k}^{+}\right)$and $R=R\left(t_{k}\right)$ ) then

$$
\begin{aligned}
P\left(t_{k+1}^{-}\right) & >\left(\frac{P+R}{R}\right) \frac{P R}{P+R}+\frac{\sigma^{2}}{2 a}\left(\frac{P R}{R}-1\right) \\
& =P\left(t_{k}^{-}\right)\left(1+\frac{\sigma^{2}}{2 a R}\right)-\frac{\sigma^{2}}{2 a} \\
& =P\left(t_{k}^{-}\right)\left(1+\frac{\sigma^{2}}{4 a R}\right)+P\left(t_{k}^{-}\right) \frac{\sigma^{2}}{4 a R}-\frac{\sigma^{2}}{2 a} \\
& >P\left(t_{k}^{-}\right)\left(1+\frac{\sigma^{2}}{4 a R}\right) \text { for } P\left(t_{k}^{-}\right)>\max [2 R, a]
\end{aligned}
$$

Hence the covariance grows at a rate greater than unity.

If we choose the resolution to be some fraction of the covariance, $R=\alpha P$, where $\alpha<<1$ then equation 13 becomes

$P\left(t_{k+1}^{-}\right)=e^{2 a\left(t_{k+1}-t_{k}\right)} \frac{\alpha}{1+\alpha} P\left(t_{k}^{-}\right)+\frac{\sigma^{2}}{2 a}\left(e^{2 a\left(t_{k+1}-t_{k}\right)}-1\right)$

and the covariance evolves as a geometric sequence

$$
P\left(t_{k+1}^{-}\right)=\gamma P\left(t_{k}^{-}\right)+\zeta \text {. }
$$

For $\gamma<1$ the covariance decreases and hence the target can be tracked. The expression

$$
\gamma<1 \quad \text { or } \quad e^{2 a\left(t_{k+1}-t_{k}\right)} \frac{\alpha}{1+\alpha}<1
$$

makes explicit the trade off between the sample interval $\left(t_{k+1}-t_{k}\right)$, the resolution sub-sampling $\alpha$, and the system dynamics, $a$. If the system is sufficiently slow for the time scale used then we can pick a resolution to ensure tracking. 